\title{
Dialectical Situations and Argument Analysis
}

\author{
JAMES B. FREEMAN Hunter College
}

One term which has recently gained much currency in informal logic/critical thinking cricles is "dialectical." A dialectical process or dialectical procedure involves an exchange, typically between two persons, where one propounds some claim and the other tries to draw out a defense, an argument for it from the claimant. Such a situation could be internalized, someone reasoning with himself over an issue, or we could imagine more than two people involved, but the exchange between two persons, a questioner and an answerer, a challenger and a respondent, is paradigmatic. We call these basic dialectical situations. Since arguments are generated in dialectical situations, various methods of argument analysis, in particular tree diagrams, are appropriate for investigating the "products" of dialectical exchanges. But there is a more intimate connection between dialectical situations and argument analysis. Certain paradigm questions which may arise are central to motivating a particularly interesting tree system for argument analysis.

Tree diagramming has gained wide currency in teaching informal logic, being included in a number of critical thinking texts. Beginning with Monroe Beardsley's Practical Logic [2] and Thinking Straight [3], continuing with Stephen Thomas's Practical Reasoning in Natural Language [11], and now with many other texts, 1 we have a method of picturing the structure of an argument by representing the component statements with encircled numbers and the support one or more statements give a conclusion by arrows, pointing downward to the conclusion. This is the standard method of argument diagramming. Independently of this, Steven Toulmin in The Uses of Argument [12] (and with Richard Rieke and Allan Janik in An Introduction to Reasoning [13]) has presented a rather different way of displaying argument structure, one which lends itself to dialectical considerations and developments. Both these methods have merit, and I believe need to be synthesized to get an adequate model of argument structure. In this paper we present such a synthesis, motivated by four paradigm questions, which we call the basic dialectical questions. We shall refer to the answerer in the basic dialectical situation as the respondent and the questioner as the challenger.2

The challenger's purpose in the basic dialectical situation is primarily positive. He does not want to silence the respondent with negative criticism, but rather to get him to present an argument for his views and to elaborate that argument in various ways. His questions, then, will be argument prompting or argument building. The structures of the resulting arguments will differ, depending on the questions asked. The simplest argument consists of exactly one premise and exactly one conclusion. Such an argument arises in a dialectical situation when our respondent puts forward an assertion, our challenger counters by asking "Why?", and our claimant responds with a statement intended as a premise for the original claim, now the conclusion. The question of argument structure becomes interesting when there are at least three component statements in the argument. The standard method of tree diagramming distinguishes four types of argument structure-convergent, serial, linked, and divergent. In an argument with divergent structure, two or more conclusions are supported by the same premise. Such arguments would arise in a dialectical situation where the respondent made two claims and gave the same 
premise when our challenger asked why each claim was true. Hence nothing new is needed to motivate this structure beyond the fundamental dialectical question "Why?". The other three structures, however, do involve further questions, three of the four basic dialectical questions. Imagine that our respondent has just given one reason for a claim. At this stage in the exchange, our challenger could react by asking first:

\section{Can you give me another reason?}

This does not question or reject the reason given. The challenger is simply asking for further evidence. If our respondent answers with a further, independent premise, the resulting argument has convergent structure, whose diagram is familiar from many texts.

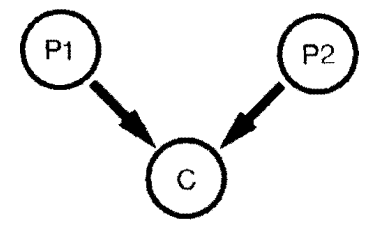

What our discussion shows is that the first dialectical question motivates convergent argument structure. By asking this question, our challenger is trying to draw out an argument with convergent structure from the respondent.

When we say that two premises are independent of each other, we mean that we do not need either premise to see why the other is relevant to the conclusion. Each separately, independently gives some evidence for the conclusion. For example, in the argument

(1) The incumbent President will be re-elected because

(2) He is a popular figure and

(3) The economy is doing well, each of (2) and (3) support (1) independently. We do not need (2) to see that (3) gives a reason for (1), or (3) to see why (2) gives a reason for (1). We shall say more about this later when we contrast convergent and linked structure and when we discuss modalities.
The second dialectical question is:

2. Why is that (i.e. the reason just given) true?

Here the challenger is not asking for more evidence, another reason to support the conclusion directly. Rather he is asking for evidence to support some premise just given. If the premise were true, it might give very strong grounds for accepting the conclusion. But why should we accept that premise? This question motivates another very familiar argument structure, serial.

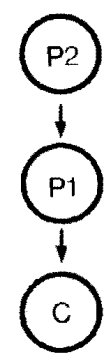

The third dialectical question is:

3. Why is that relevant (i.e. why is the reason given relevant to the basic claim being argued)?

Here is a sample dialectical situation where the third question might arise:

$\mathrm{R}$ : Jones' car uses too much gas.

C: Why?

$R$ : It is a 1968 model.

Now the respondent's answer is relevant to his claim. But it may not be apparent to everyone, including our challenger. We need to know something about cars made in 1968. Hence

C: What does being a 1968 car have to do with gas use? (i.e. Why is that relevant?)

might very well be our challenger's rejoinder. The answer

R: Cars made around 1968 are far less fuel efficient than other models and if a car is less fuel efficient than some other model, it uses too much gas

should satisfactorily explain why being a 1968 model car is relevant to using too much gas. The argument structure motivated by this question is again familiar from standard texts. Where 
(P1) is the first reason given and (P2) is the statement explaining why (P1) is relevant to the conclusion $(C)$, the structure is linked:

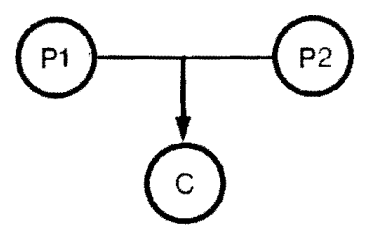

Although this structure is familiar, some texts use it to indicate another way in which statements may combine to support a conclusion, which we shall consider momentarily. For now, we should make it plain that our use of linked structure is exclusively tied to this question of relevance. Two (or more) statements should be linked together when we need to take them together or they are intended to be taken together to see why we have a relevant reason for the conclusion. Classical syllogisms, for example, are paradigm cases of linked argument structure. In linked struture, the two (or more) premises linked together depend on each other to constitute a relevant reason for the conclusion. They do not, as in convergent argument structure, constitute two reasons independently supporting the conclusion. Rather we have one reason broken over at least two statements.

Before considering the fourth dialectical question, we should recognize the critical component in the three basic dialectical questions we have already examined. We do this because having the critical component in mind makes the fourth dialectical question more understandable. When a challenger asks for another reason, ordinarily he is not motivated by idle curiosity. He does not want to see just that the respondent can produce another reason. Rather he is motivated by the feeling that the reason given, although giving some plausibility to the conclusion, may not be strong enough to produce a really convincing case for the respondent's basic claim. So lying behind this first basic dialectical question is a critical consideration or question:
Are enough reasons given or are the reasons strong enough to establish the conclusion?

A negative answer to this critical question, alternatively our respondent's inability to answer the first dialectical question, would constitute grounds for a negative evaluation of the argument. If we want to use labels, the argument would be an instance of the hasty conclusion fallacy.

Similarly, the second dialectical question is ordinarily motivated by critical concerns. Our challenger will not usually ask for some justification for a premise merely to prolong the discussion, merely to see whether the respondent can give an answer. Rather, he is prompted by another critical question:

\section{Are the premises true or warranted?}

A negative answer to this question or failure to answer the second dialectical question leaves the argument open to another negative evaluation. It involves either a false or questionable premise. Again, when a challenger asks the third dialectical question, the critical consideration:

\section{Is the reason relevant?}

prompts his query. Failure to properly answer these questions indicates the argument involves the fallacy of irrelevant reason. Hence, the three dialectical questions, although positive, tending to draw out the respondent to further elaborate his argument, are critically motivated. They are motivated by potential weaknesses or failure in an argument which the respondent should shore up before regarding his case as complete. This is also the case for the fourth dialectical question, which we shall see after we introduce one further concept.

When a person puts forward one or more reasons to support a conclusion, he or she is claiming that these reasons create a presumption for the conclusion. Hence to argue

(1) Martina is highly motivated.

(2) She also has good scholastic 
aptitude. Hence

(3) She will do well in college. as opposed to

(1) Martina is highly motivated.

(2) She also has good scholastic aptitude. Hence, presumably,

(3) She will do well in college is to say the same thing. The addition of the word "presumably" does not add anything to the cognitive content of the argument. However, it does add a new structural component. The word "presumably" is a modality. A modality is not a component either of the premises or the conclusion of an argument. Rather it serves to modify the claim that the premises support the conclusion. It is an additional element in the argument. Steven Toulmin advocates explicitly representing this element in the diagram of the argument. Adapting Toulmin's procedure, we represent modalities by enclosing the modal word in a box, interposed between the premises and conclusion. Thus the second version of the above argument is diagrammed this way:

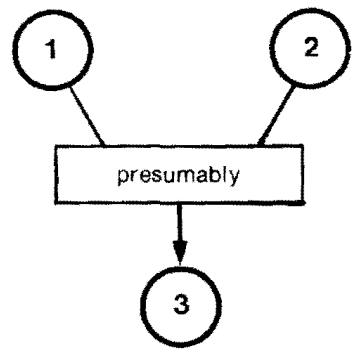

There are a number of advantages to allowing for the explicit representation of modalities in arguments. First, although modalities may frequently be written infixed in the conclusion, they are not strictly part of the conclusion but of the connecting material between premises and conclusion. They serve to make a metalinguistic claim about how strongly the premises support the conclusion. Thus they are elements distinct from premises and conclusions, and our representation technique enables us to display this perspicuously.

Secondly, as noted above, there are two ways in which premises may combine to support conclusions. Linked argument structure indicates that premises must be taken together to constitute a relevant reason for the conclusion. Now consider our above argument. The two premises independently support the conclusion. But in assessing the overall strength of the argument, we do not look at these two reasons separately, as if we had two totally separate arguments here, but will combine the weight of these two reasons in assessing how strong a case we have for the conclusion. The structural representation of modalities allows us to display this modal connection, modal "linkage" if you will, of statements very perspicuously. Our diagram indicates that although (1) and (2) are separate, distinct, independent reasons for (3), their weight is combined in claiming that our premises give us a presumption for the conclusion. Relevance linkage and modal linkage, thus, are two different ways coften amalgamated in the standard argument diagramming technique and represented as linked arguments) for premises to combine in supporting a conclusion. Our structural resources allow us to give each a distinct representation.

There is another reason for explicitly representing modalities in our argument diagrams, but we must postpone discussing it until we have made a few further remarks about modalities and have introduced the fourth dialectical question. There are many other modalities besides "presumably": "it is strongly presumable that," "probably," "likely," "very likely," "certain beyond a reasonable shadow of a doubt," "it is morally certain that" - all these expressions are modalities. They all serve to make claims, although of varying strength, about the degree of support premises may give conclusions. We may also call them inductive modalities, since although some of these modalities claim that the premises give a very high degree of support to the conclusion, none claim that the premises guarantee that the conclusion is true, that if the premises are true, the conclusion must be true also. 
This is a deductive claim, expressed by such modalities as "necessarily" and "it must be the case that."

Any of these modalities may appear in arguments, and their presence in some circumstances may prompt our challenger to ask the fourth dialectical question:

4. Why does that (i.e. the premises given whose weight is being combined in the modality) make you so sure?

Why would our challenger ask this question? Again, although idle curiosity is abstractly possible, a critical motivation is more genuine: Our challenger questions:

Are the reasons strong enough to establish the conclusion with the strength claimed?

Given the premises, this may not be obvious. For example, suppose in our above argument we replace "presumably" by "certainly." So we are arguing that Martina's high motivation and good scholastic aptitude make it certain that she will do well in college. Now "certainly" is a very strong modality. Although we have two good, strong reasons here, do they really make it certain that Martina will do well? Are we justified in using such a strong modality? This is a legitimate critical question. A negative answer, or inability to answer the fourth dialectical question, would also be grounds for a negative evaluation of the argument. We again have a fallacy of hasty conclusion.

How might our respondent answer this fourth dialectical question? That will depend on the particular circumstances of the argument. If we are dealing with a deductive modality, he must argue that the premises do provide conclusive grounds for the conclusion. This may involve an exercise in formal logic, and so here the techniques of modern symbolic logic may be useful. It might also involve some sort of conceptual analysis.

With inductive modalities, a far more interesting-dialectically interesting case arises. For the purposes of il- lustration, let us grant that although "certainly" may express a deductive modality, it need not on all occasions. We shall take it as expressing an inductive claim here, albeit a claim of very strong support. Our challenger may feel that Martina's motivation and scholastic aptitude do not make it certain that she will do well in college because he foresees conditions possibly holding which would undercut this argument. If these conditions hold, then the premises may be true and the conclusion false. Recognizing their possibility should make the conclusion less certain vis-a-vis the premises. For example, it is possible that Martina has just had a nervous breakdown or that she has become romantically involved. Both could have an adverse effect on her academic performance. Recognizing these possibilities should lead us to question the strength claimed by "certainly." Again borrowing terminology from Steven Toulmin, we call these undercutting conditions rebuttals. Toulmin defines rebuttals as "the extraordinary or exceptional circumstances that might undermine the force of the supporting arguments."3 We understand rebuttals more broadly as any undermining condition, not necessarily extraordinary or exceptional. Here is another example:

(1) Mrs. Wilson's probated will leaves all her money to charity, cutting off her daughter with only $\$ 1.00$ from her estate. Presumably

(2) $\$ 1.00$ is all Mrs. Wilson's daughter will get from her mother's estate.

Clearly, the premise creates a presumption for the conclusion. But suppose the daughter could prove that her mother was mentally incompetent when she made the will, or the laws of the state in which the will was made prohibited the disinheriting of children, or that a later will invalidating this one could be found. Then the force of the presumption would be completely 
undercut.

How should we represent rebuttals structurally in our diagram? Here again, we adapt some notation of Toulmin's. The rebutting conditions, in effect, qualify how strongly the premises support the conclusion. We show this by writing out the rebutting conditions, enclosing them in a box, and then attaching that box to the box containing the modality. Thus our argument about Mrs. Wilson is represented as:

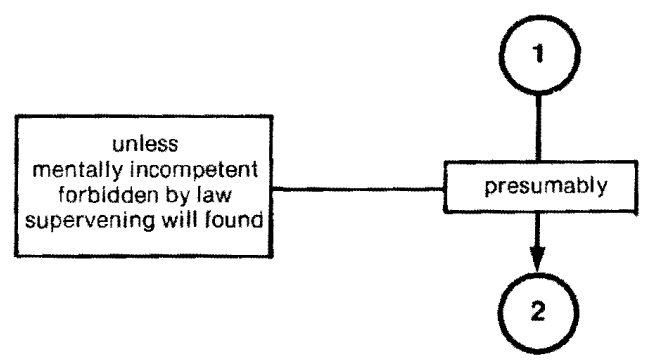

The argument about Martina looks like this:

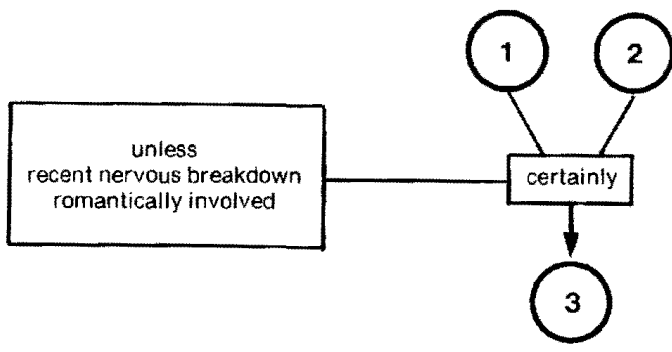

Rebuttals differ in the seriousness of the challenge they pose to arguments, depending on whether the burden of proof is on the challenger to show that the rebuttals actually operate or on the respondent to show that they do not. This, in turn, depends on how likely it is that the rebuttal holds. Rebuttals as Toulmin characterizes them, extraordinary or exceptional circumstances, presumably have low probability. If we say that certain premises create a presumption for the conclusion, or make our conclusion likely to a certain degree, this statement is still true even when such rebuttals are presented. Their mention clarifies the modality, making it less vague, more precise under exactly what conditions the premises create a presumption for the conclusion. We could regard the < modality, rebuttal $>$ pair as a complex modality indicating how strongly the premises support the conclusion. This also holds true whenever the burden of proof is on the challenger to show that the rebuttal holds. However, if there is a significant probability that the mentioned rebuttal holds, then the claim that our premises create a presumption for the conclusion or create it with a certain degree of strength is called into question. Without showing that this rebuttal does not hold, use of the modality is no longer correct. Without the rebuttal component in the <modality, rebuttal $>$ pair, we have a fault in the argument.

When our challenger asks the fourth dialectical question because he has one or more rebuttals in mind, it is presumably because he is either willing to undertake the burden of proof to show that his rebuttal operates in this case, or he recognizes that when once introduced, the burden of proof would be on the respondent to show that the rebuttals were false. In either case, the argumentative exchange can be extended, but to adequately represent the structure, we need to introduce further devices. Suppose the burden of proof is on the challenger, who rises to this challenge. At this point, we actually have two arguments going, the argument of our original respondent and the attacking argument of our challenger. In diagramming such a situation, we should diagram the challenger's argument on one side. ${ }^{4}$ The component statements in this argument should also be assigned numbers. Hence the conclusion appears at least twice in the diagram, as conclusion of the challenger's argument, where it is numbered, and as a rebuttal in the original argument, where it is written out in the rebuttal box. To show that these two representations indicate the same thing, we should write the number of the conclusion next to the rebuttal in the box. For example, if in the dialectical situation above discussing Mrs. Wilson, the challenger knew she had been suffering from 
Altzheimer's disease for the last ten years before she died, her condition progressively worsening, and that she had made the will only a year before she died, that would create a strong presumption that she was mentally incompetent. Assigning (3) to the statement that Mrs. Wilson was mentally incompetent, (4) to the statement about her suffering from Altzheimer's disease, and (5) to the statement that she had made her will only a year before she died, the diagram of the two arguments in the situation should look like this:
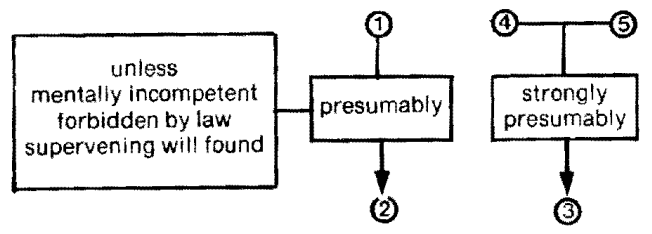

If the burden of proof is on the respondent, he may answer with a counterrebuttal. This could be either a statement that the rebutting condition does not hold, a counterrebuttal proper, or an argument to that effect. Counterrebuttals are in effect a type of premise. They support the conclusion, but not by giving evidence which directly supports it but rather by showing that certain possible rebuttals are not operative. Hence, we structurally represent counterrebuttals by assigning numbers to them, as we do to the other premises and conclusions in the argument, and then drawing downward directed arrows from these numbers to the rebuttal box. This shows that these statements support the conclusion, but support it by ruling out rebuttals. Supposing then that our respondent is prepared to claim that Mrs. Wilson was not mentally incompetent (3), that disinheriting her daughter was not forbidden by law (4), and that no supervening will could be found (5), her argument that Mrs. Wilson's daughter will get only $\$ 1.00$ from her mother's estate may be diagrammed this way:

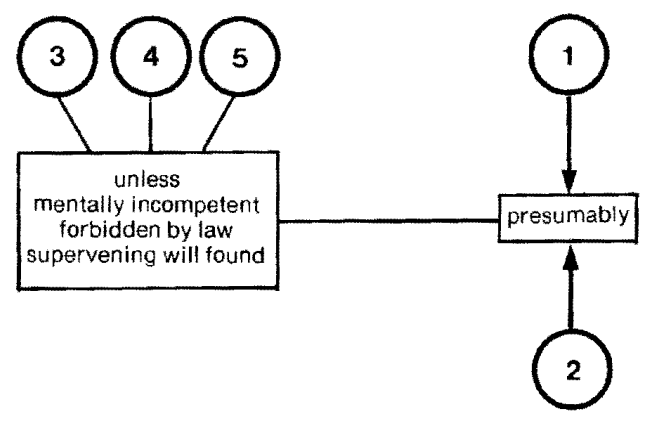

If our respondent has presented a whole counterrebutting argument for either (3), (4), or (5), we can represent that by a tree structure, the whole diagram for this argument appearing above the counterrebuttal box.

If our challenger has presented an entire argument to show his rebutting condition holds, our respondent's counterrebuttal may consist of an attack on that argument, attempting to show that it is not cogent. In this case, the counterrebuttal is rather complex, consisting of an argument that another argument does not work. How may we diagram this? The challenger's argument for his rebuttal should be diagrammed, enclosed in a box, and crossed out to represent the claim that this argument is not cogent. Our respondent's argument for noncogency should be diagrammed above this box, with downward directed arrows indicating that it supports rejecting the rebutting argument. To continue our example, although our challenger may claim that Mrs. Wilson made her will only a year before she died, suppose our respondent knows that she made the will eleven years before she died because of the date on her will. That would attack the truth of one premise in the argument and so cause the case it makes for Mrs. Wilson's being mentally incompetent to collapse. Where (6) numbers the statement that Mrs. Wilson made her will eleven years before she died and (7) the statement about the date on the will, and (3), (4), (5) are as in the rebutting argument above, this counterrebutting train of reasoning should look like this: 


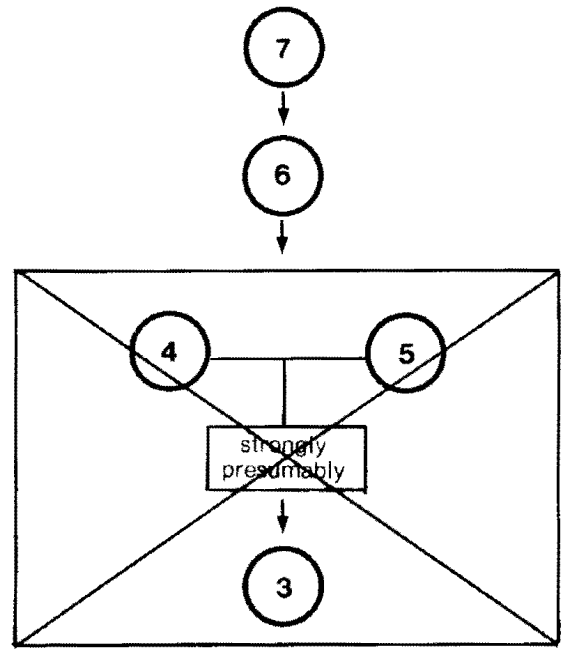

We can now either enter this whole diagram in our main argument as a counterrebuttal, or we can represent it on the side, assigning the Roman numeral $\mid$ to the rebutting argument. The counterrebuttal then says that (I) is non-operative, and we represent this by writing ( 1 ) in a circle crossed out, placed in the position of a counterrebuttal, as we do below.

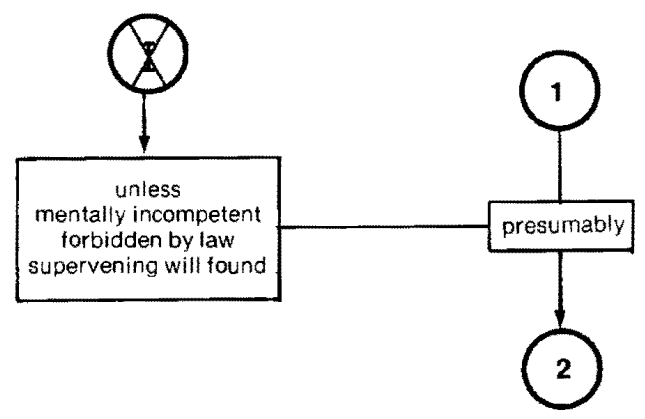

Of course, we can imagine our challenger presenting an argument to counter this counterrebuttal, a countercounterrebuttal, if you will, and our respondent replying with a further argument. Although this may sound complicated at this point, our diagramming system allows us to structure out this situation. The resources we have to construct diagrams are rich enough to allow this structural representation. By doing so, we presumably can get a handle on how a whole argumentative passage hangs together, how a dialectical exchange is structured, how the arguments and counterarguments fit together.

Developing a complete system of argument diagramming requires considering one further issue: argument strategy. Counterrebuttals, as opposed to the other premises we have considered, do not give evidence directly for a conclusion but rule out possible undercutting conditions. We may see two strategies here for defending a conclusion-giving direct evidence and counterrebuttals. We may also distinguish other strategies which may occur within either direct or counterrebutting strategy. Instead of offering a single statement as a reason why some claim is true, we may offer the fact that we can argue from one statement to another. This occurs in conditional proof strategy and reductio ad absurdum arguments. To defend why a conditional statement is true, we may show that we can argue from antecedent to consequent. Alternatively, to show a statement true, we may assume its opposite and argue to a statement recognized false. Since these responses would be prompted by such dialectical questions as "Why?", "Can you give me another reason?", or "How do you know that is true?", motivating them does not require further dialectical questions. We may represent arguments involving conditional and reductio strategies by enclosing the subarguments involving assumed statements in boxes, with a downward directed arrow from the box to the statement being supported. ${ }^{5}$ We shall not develop this, since the details of these strategies do not affect our central concerns. We should stress here that as the first three basic dialectical questions motivated convergent, serial, and linked argument structure, so the fourth dialectical question motivates the introduction of modalities, rebuttals, and counterrebuttals. Having these concepts at hand enables us to structurally represent arguments, and the fourth dialectical question motivates why these concepts may be useful and important in argument analysis.

We have seen how each type of argu- 
ment structure is motivated by a dialectical question. We may now ask what is the purpose of developing this system of argument diagramming motivated by the four basic dialectical questions? The presence of tree diagramming techniques in many texts suggests their utility in argument analysis. This utility is easily appreciated. How can one understand an argument unless he or she can distinguish premises from conclusions? How can one understand an argumentative passage unless he or she can distinguish the various strands in an argument which may converge to support a conclusion and how those strands are composed? Diagramming an argument should sharpen our understanding of it. But we may look at the primary arena for argument as the dialectical situation, where there is an exchange between two or more persons. Arguments presented in prose may be viewed as abstractions from this primary mode of argumentation. Motivating our system of argument diagramming with the basic dialectical questions connects the whole issue of argument structure and analysis with this primary field for arguments. There is a distinct degree of abstraction to constructing argument diagrams. By tying the tree structure to concrete dialectical issues, the point of analysing arguments with tree diagrams should have intuitive motivation.

In addition, having the four basic dialectical questions in mind and the critical questions which underly them should aid in criticizing arguments once we see how they are structured. Can a rebuttal which significantly undercuts the argument be framed and defended? Can we question the amount of support, the truth of the support, or its relevance to the conclusion? Our diagramming procedure and its motivating questions let us focus on these issues. Furthermore, these questions should aid us in constructing arguments. If we can imagine what dialectical questions might be asked, what rebuttals might be presented against an argument we are developing, we should see what sort of case we must present to properly establish the conclusion. By anticipating and answering these questions, our ability to compose cogent arguments may be enhanced. In addition, being able to diagram arguments should aid in organizing arguments of our own

The basic dialectical questions have a further advantage. Our presentation of dialectical situations is admittedly abstract. Our participants in these situations, our challengers and respondents, have been rather faceless individuals. We have considered how they could make claims and give defenses, ask questions and wage counterattacks, without saying anything about them, about how their particular contribution to the dialectical situation expresses their assumptions, beliefs, attitudes, and values. But it seems just a single step, and a very natural step, to regard each of the participants in a dialectical situation as bringing a whole point of view with him into the situation. I believe we can be more specific here. Psychologists have traced the development of a person's self-image, what he thinks of himself, how he regards himself. Social psychologists who are concerned with propaganda and students of rhetoric introduce the concept of a world image or image system. This is a system of beliefs, attitudes, and values which constitutes a picture of how the world is ${ }^{6}$ We may say that each person develops a $<$ self, world $>$ image system with which he interprets the experiences and in particular the messages he continually receives. A person's mind is not a tabula rasa on which incoming messages are inscribed. Rather a message must interact with this whole image system. Our picture of the critical challenger may very well represent an ideal of dialectical rationality, dispassionately seeking to weigh a case on its merits. A person ordinarily will have a significant emotional investment in this $<$ self, world $>$ image system, for it tells him who he is, who or what others are, what is good, what is evil. Al- 
though the image system has a profound effect on a person's beliefs and values, ordinarily it is not an altogether conscious system. Parts of the system may only be at the background of awareness; parts may be completely unconscious.

A person's < self, world $>$ image system may easily exert a profound influence on his participation in a dialectical situation. Consider the role of respondent. Clearly someone may hold a certain view because it fits in with his image system. The beliefs and valuations constituting the image system render support to the view. He may see certain evidence as relevant to a claim, or as supporting it with a certain amount of weight, again because of background assumptions in his image system - assumptions concerning general human behavior, natural regularities, or value hierarchies. Now just because a factual belief or value judgment is included in the image system does not mean that it is true. On the contrary, it could be distinctly questionable. But as long as the belief is at most at the background of consciousness, it will not be critically questioned. But our basic dialectical questions could be a way of bringing these assumptions to the light of full consciousness where they can be critically examined and evaluated. Just the very question "Why?" requires the respondent to formulate explicitly a reason for his claim. Once that is formulated, the questions "Can you give me another reason?" "Why is that true?", "Why is that relevant?", "Why does that make you so sure?" lead the respondent to reflect critically on his claim, formulating explicitly other premises, especially general statements of fact or value which may have been merely assumed as part of the <self, world> image system.

Being asked to think critically about these assumptions should be a liberating experience in several ways. First, if we can produce reasons for our assumptions, can argue for them plausibly, then we may realize that the beliefs and values we hold are not a matter of mere opinion, but may stand on their own merits. Our views are not merely a matter of subjective taste, but can be defended, where the defense has some degree of logical compulsion, requiring our challenger to agree with us or to produce some challenge to our argument. 7 Thus our dialectical procedure should be liberating from the subjectivism and relativism seen as so rampant today. On the other hand, the dialectical procedure should liberate us from dogmatism, or may serve as an occasion for shaking our dogmatism. Having our assumptions challenged may lead us to reject some and to regard others as only plausible, not as absolutely true. But this might result in an attitude of tentativeness and detachment towards our < self, world $>$ image system. Now this attitude of detachment goes straight to the heart of liberation. If we can accept our image system with an air of tentativeness, then the emotional needs and desires, many of them irrational, which may have fostered the development of the system will no longer be dominant, exerting a controlling influence on our beliefs and values. We may still use our image system pragmatically to interpret the world, but we shall be aware of what we are doing and of the tentativeness of our interpretations.

There is a further aspect to the liberating nature of the dialectical process. Consider the challenger. By drawing out the respondent through asking him the basic dialectical questions, our challenger is trying as best he can to enter into the respondent's point of view, his <self, world> image system, even if the challenger finds the constituent beliefs and values egothreatening. Furthermore, our respondent's anticipating a challenger's questions, in particular anticipating how he might argue for a rebuttal, in effect switching roles with the challenger and letting him become respondent defending his rebuttal, is to attempt to enter into the challenger's $<$ self, world > image system. The dialectical exchange allows for this mutual 
interpenetration of image systems. But, as Richard Paul has pointed out, "Learning to listen to and read (with" out distortion) lines of reasoning whose possible truth we egocentrically wish to rule out, is an essential experience, indeed the mother's milk, of educational development.... When outside the purely technical, part of the dues we must pay to justify rational confidence is empathy into the strongest case that can be made against our conclusion."8 Our dialectical questions provide us with a key for entering into the points of view of others, and not to enter only in an attempt to find out where the other person is coming from but to critically assess his view and appropriate what it contains of value for appraising and justifying our own claims. But to appreciate the views of others, to be critically open to them, is this not the hallmark of a liberally educated person? Hence our dialectical questions not only motivate a system of argument analysis and facilitate argument critique and construction, they aid in developing the attitudes at the heart of being a rational person, at the heart of liberal education. It is for all these reasons that we see dialectical situations and argument analysis as important tools in the informal logic/critical thinking enterprise.

\section{Notes}

1. See for example Vincent Barry's Invitation to Critical Thinking [1], John Eric Nolt's Possible Worlds and Imagination: An Introduction to Informal Logic [6], and Ralph $\mathrm{H}$. Johnson and J. Anthony Blair's Logical Selt-Defense [5]. Michael Scriven's Reasoning [9], a pioneer informal logic text, uses tree diagramming, and the latest edition of Irving $M$. Copi's Introduction to Logic [4] includes sections on tree diagramming.

2. Those familiar with Carl Wellman's Challenge and Response: Justification in Ethics [14] will see how that book has provided a seminal motif for our thinking here.

3. [13], p. 95.

4. The idea of diagramming a rebutting argument on the side is suggested by Michael Scriven in Reasoning [9].

5. Stephen N. Thomas in [11] suggests this way of handling conditional and reductio ad absurdum arguments. As we have seen, it can be adapted for certain counterrebutting arguments where a whole argument is attacked.

6. The image system is discussed by J. Michael Sproule in Argument: Language and Its Influence [10], Chapter Seven.

7. Following Robert Pinto in [8], we may say that an argument creates a presumption for our conclusion, where "A proposition or statement has the status or a presumption at a given juncture of an interchange if and only if at that juncture any party who refuses to concede it is obliged to present an argument against it - that is to say, is obliged either to concede it or to make a case against it." ([8], p. 17)

8. [7], pp. 21, 26.

\section{Bibliography}

[1] Barry, Vincent. Invitation to Critical Thinking. New York; Holt, Rinehart and Winston, 1984.

[2] Beardsley, Monroe. Practical Logic. Englewood Cliffs, N. J.; Prentice-Hall, Inc., 1950.

[3] Thinking Straight. Fourth Edition. Englewood Cliffs, N.J.; Prentice-Hall, Inc., 1975.

[4] Copi, Irving $M$. Introduction to Logic. Sixth Edition. New York; Macmillan Publishing Co., Inc., 1982. 
[5] Johnson, Ralph H. and Blair, J. Anthony. Logical Self-Defense. Toronto; McGraw-Hill Ryerson, 1977.

[6] Nolt, John Eric. Possible Worlds and Imagination: An Introduction to Informal Logic. New York; McCraw-Hill Book Company, 1984

[7] Paul, Richard. "The Concept of Critical Thinking: An Analysis, A Global Strategy, and Plea for Emancipatory Reason." Forthcoming.

[8] Pinto, R. C. "Dialectic and the Structure of Argument." Informal Logic vi.1 (1984), 16-20.

[9] Scriven, Michael. Reasoning. New York; McGraw-Hill Book Company, 1976.

[10] Sproule, J. Michael. Argument: Language and Its Influence. New York; McGraw-Hill Book
Company, 1980.

[11] Thomas, Stephen N. Practical Reasoning in Natural Language. Second Edition. Englewood Cliffs, N. J.; Prentice-Hall, Inc., 1981.

[12] Toulmin, Steven. The Uses of Argument. Cambridge; Cambridge University Press, 1958.

[13] Toulmin, Steven, Rieke, Richard, and Janik, Allan. An Introduction to Reasoning. Second Edition. New York; Macmillan Publishing Co., Inc., 1984.

[14] Wellman, Carl. Challenge and Response: Justification in Ethics. Carbondale and Edwardsville; Southern Illinois University Press, 1971.

Professor James B. Freeman, Department of Philosophy, Hunter College, 695 Park Avenue, New York NY 10021 